\section{PHYSICS CONTRIBUTIONS}

AN APPLICATION OF $200 \mathrm{KV}$ TO ENGINEERING MLATERIALS

\section{J. Beyer}

Dept. of Mechanical Engineering, Section Materials Science, Twente University of Technology, Enschede, The Netherlands

The relation between microstructure and relevant mechanical or physical properties is of crucial importance for the understanding of the behaviour of materials in practice. Optical and electron microscopy are the appropriate instruments to reveal the microstructure. The combination of the CTEM with an EDS detector, energy loss spectrometer, image analyzer and the possibilities for STEM and SEM converted this instrument to comprehensive analytical tool for the material scientist. The development of "low cost" transmission microscopes working at higher voltages than $100 \mathrm{kV}$ opens new possibilities for the investigation especially of the complex microstructures in engineering materials like steels. After summarizing the arcuments for the application of higher voltages, a more detailed description will be given of the penetration power of the $200 \mathrm{kV}$ JEOL instrument installed recently in our $1 \mathrm{ab}$.

Illustrations from three research projects in progress will be given:

-Dual phase steels have often a more complex structure than is suggested by the name. Ferrite, martensite, bainite, retained austenite and often perlite can coexist, together with different types of carbides, nitrides and other inclusions.l In such a case it is important to be able to observe large areas at low magnification, before selecting the relevant phases for more detailed investigation.

- -High alloy chromium steel (10\% Cr) is used as a heat-resistant valve steel in practice. A martensitic transformation $\mathrm{M}_{23} \mathrm{C}_{6} \rightarrow \mathrm{M}_{7} \mathrm{C}_{3}$ can occur in the $\mathrm{Cr}$-carbide present in this steel which has a detrimental effect on the mechanical properties. This transformation can only be investigated in relatively thick specimens. In the preparation of thin foils the relatively thick carbides would drop out of the thin areas. If on the other hand the carbides are very thin, the high stresses produced by the transformation are relaxed and the structure can change. - Co-Cr alloy is developed as a new information carrier, with high bit density, for vertical or perpendicular recording. 2 The magnetization is perpendicular to the sputtered film. However, the crystal size C $0.1 \mu$ in a film of $1 \mu$ thickness decreases with film thickness, resulting in higher bit density but a higher noise level. The growth kinetics and uniformity of crystal size are investigated.

1. Formable HSLA and dual-phase steels (ed. A.T. Davenport, Proc. Metallurgical Society of AIME, 1977).

2. T. Wielinga and J. C. Lodder, IEEE trans. on magnetics, to be published (1981).

\section{IS THERE AN ELECTRON MICROSCOPE FOR} GEOLOGICAL NEEDS?

J. N. Boland

Instituut voor Aardwetenschappen,
Vakgroep Structurele en Toegepaste
Geologie, Budapestlaan 4, Postbus
$80.021,3508$ TA Utrecht, Netherlands

It may be presumptuous to think that geologists make more specialized yet broader-based requests of their electron microscope than other researchers. However, I have reasons to think that this is the case.

Unlike most crystalline materials studied by materials scientists, minerals present us with a genuine problem of not knowing precisely their mode of formation and subsequent history of development. For many geologists a solution to this problem is the whole business of their study. This problematical situation can be described best in a study of the amphibole group of minerals. Amphibole may form in any of the following ways:

a) normal crystal growth from a liquid (magma) during solidification;

b) during the degradation of preexisting minerals because the pressure, $P$, and temperature, $T$, have decreased (probably accompanied by a chemical change, $x$, in the system)--retrograde metamorphism;

c) during "solid"-phase crystallization (precipitation) in a rock because $\mathrm{P}, \mathrm{T}$ and $\mathrm{X}$ have changed in the direction of amphibole stability--prograde metamorphism.

One must add to this repertoire the interfering effects of deformation which may be regional, local or submicroscopic. The non-uniqueness of formation imposes many problems in deciphering the origin of defect structures such as exsolution lamellae (precipitated phases) or dislocation substructures.

The normal ploy of consulting the equilibrium diagram to determine phase stabilities has limited value. This is especially true for the amphibole minerals in which the phase relationships are 